\title{
Übungen in der medizinpsychologischen Lehre
}

\author{
Friederike Kendel, Götz, Fabry, Swetlana Philipp, Jens-Stefan Geier \& Katrin Rockenbauch
}

\section{Hintergrund}

Seit 1970 ist die Medizinische Psychologie ein fester Bestandteil der Approbationsordnung für Ärzte (ÄAppO) und ein Pflichtfach im Medizinstudium. Ein wichtiger Teil des Curriculums sind die Themen Arzt-Patient-Beziehung und Kommunikation (Petersen et al. 2006).

Vermittelt werden jedoch auch theoretische Grundlagen der Psychologie, welche Themen wie Lernen, Kognition, Emotion, Motivation, Persönlichkeit, Entwicklung und Methoden umfassen. Bei allen Themengebieten soll der Brückenschlag zu psychologischen Aspekten von Krankheit und Gesundheit gelingen: sowohl in der theoretischen Fundierung als auch in den klinischen Bezügen. Als Beispiel sei das Thema „Emotion“ genannt: Emotionen spielen im klinischen Alltag eine bedeutende Rolle. Eine Grundlage für das Verständnis von Emotionen bildet zunächst die Kenntnis von Basisemotionen und (neuro-)psychologischen Theorien der Emotionsentstehung. Ausgehend davon befasst sich die Medizinische Psychologie mit Emotionen in der klinischen Praxis. Betrachtet werden sowohl Emotionen in ihrer durchschnittlichen Ausprägung als auch affektive Störungen wie Depression oder Angststörungen.

Die oben aufgeführten theoretischen Grundlagen sind auch Bestandteile eines klassischen Psychologiestudiums. Während jedoch im Psychologiestudium jedes Thema, sei es Stressverarbeitung, Methoden, Entwicklung oder psychobiologische Grundlagen, während eines ganzen Semesters vertieft wird, steht derzeit in den Regelstudiengängen Humanmedizin für jedes Thema nicht wesentlich mehr als eine Doppelstunde zur Verfügung. Die Dozierenden sehen sich also vor der Herausforderung, grundlegende Theorien in einem Bruchteil der Zeit abhandeln zu müssen. Angesichts einer solchen Zeitknappheit besteht die Gefahr, dass komplexe Theorien des menschlichen Erlebens und Verhaltens als allzu theoretisch, im schlimmsten Fall als unwesentlich oder sogar banal empfunden werden.

Ein wirklicher Ausweg aus diesem Dilemma scheint nicht möglich. Allerdings können Übungen und prägnante Beispiele aus dem klinischen Alltag dazu beitragen, das Problem der Zeitknappheit zu entschärfen.

Gute Übungen oder anschauliche Beispiele für eine Theorie können:

- das Interesse wecken und die Aufmerksamkeit auf ein bestimmtes Problem lenken.

Korrespondenzadresse: Dr. Friederike Kendel, CharitéCentrum für Humanund Gesundheitswissenschaften, Institut für Medizinische Psychologie, Charité - Universitätsmedizin Berlin, Luisenstraße 57, Berlin. Email: friederike.kendel@charite.de.
- den Transfer psychologischer Inhalte auf den klinischen Alltag erleichtern.

- das Gelernte durch die aktive Anwendung nachhaltig im Gedächtnis verankern.

Im September 2012 fand im Rahmen des Kongresses der Deutschen Gesellschaft für Medizinische Psychologie (DGMP) ein Workshop der Kommission Lehre statt, der dem Thema „Übungen in der medizinpsychologischen Lehre“ gewidmet war. Dabei wurde deutlich, dass es bereits eine Fülle bewährter Übungen zur Arzt-Patient-Beziehung und zu Kommunikation im Allgemeinen gibt. In Hinblick auf die zu lehrenden theoretischen Grundlagen wurde hingegen ein Mangel an Übungen und guten Beispielen konstatiert.

Ziel dieses Beitrages ist es deshalb, drei Übungen zur Vermittlung von theoretischen Inhalten der Medizinischen Psychologie beispielhaft vorzustellen. Der erste Beitrag zeigt, wie mit einer Übung zum Forschungsprozess (,Wie forscht man eigentlich?") zum einen Interesse geweckt werden kann und zum anderen der Wissensstand der Teilnehmer transparent wird. Der zweite Beitrag befasst sich mit Übungen, die in das Gedächtnismodell von Baddeley (2000) und Mnemotechniken einführen. Der dritte Beitrag veranschaulicht, wie Studierende durch eine eigene Erfahrung für Prozesse der sozialen Wahrnehmung sensibilisiert werden können.

\section{Literatur}

Petersen, C., Philipp, S., Rockenbauch, K., Daig, I. \& Fabry, G. (2006). Medizinpsychologische Lehre in Deutschland - eine Bestandsaufnahme. Zeitschrift für Medizinische Psychologie, 15(1), 5-10.

\section{Drei Übungen zu theoretischen Grundlagen}

\section{Methodische Grundlagen: „Der Forschungsprozess“}

\section{Katrin Rockenbauch}

Kurzbeschreibung: Die Übung zum Forschungsprozess soll die Studierenden neugierig auf das Thema „Forschung“ machen und zu einer Auseinandersetzung mit den methodischen Grundlagen anregen. Im Verlauf der gesamten Einheit werden die einzelnen Phasen des Forschungsprozesses vorgestellt und erklärt. Gleichzeitig erlaubt die Übung eine Einschätzung über den Wissensstand der Studierendengruppe zum Zeitpunkt des Seminars. 


\section{Der Forschungskreislauf}

Im Folgenden finden Sie Begrifflichkeiten, die zu einem Forschungsprozess gehören. Klären Sie für sich zunächst die Begrifflichkeiten und bringen Sie diese dann in die

Reihenfolge, die Sie einhalten würden, wenn Sie forschen würden.

Diskutieren Sie Ihre Vorschläge dann in der Gruppe und einigen sich auf einen Ablauf, den

Sie auf ein Flipchartpapier zeichnen und in der Großgruppe vorstellen.

\begin{tabular}{|c|c|}
\hline $\begin{array}{c}\text { Interpretation der } \\
\text { Ergebnisse }\end{array}$ & Menschen beobachten \\
\hline Theoriebildung & Ethikkommission anfragen \\
\hline Hypothesen bilden & Literaturrecherche \\
\hline Literaturrecherche & Hypothese falsifizieren \\
\hline Interviews führen & Daten eingeben \\
\hline Fragebogen erstellen & Interviews auswerten \\
\hline Operationalisierung & Qualitative Forschung \\
\hline Versuchsplanung & Datenauswertung \\
\hline Experiment & Untersuchungen anderer \\
\hline lesen
\end{tabular}

Abbildung 1.

\section{Lernziele}

Die Studierenden sollen...

- die einzelnen Phasen des Forschungsprozesses benennen und in ihren Grundzügen erläutern können.

- eigene Wissenslücken erkennen.

Voraussetzungen: Vor dieser Übung können einzelne Forschungsmethoden (zum Beispiel Experiment, Beobachtung, Befragung, Test) bereits behandelt worden sein bzw. die Studierenden haben sich die Unterschiede durch das Lesen kurzer Texte dazu bereits beigebracht. Dafür eignet sich die Methode „Gruppenpuzzle“ (Jürgen-Lohmann et al. 2001) sehr gut; geeignete Texte, die gleichermaßen in die Tiefe beziehungsweise in die Breite gehen finden sich zum Beispiel bei Bortz und Döring (2006).

Instruktion und Durchführung: Zunächst bekommt jede/r Seminarteilnehmer/in (bis zu 22 Teilnehmer/innen, ideal sind 16 Personen) ein Arbeitsblatt, auf dem Begriffe zum Forschungskreislauf aufgelistet sind (vergleiche Abbildung 1).
Alle Teilnehmer/innen sollen zunächst in Einzelarbeit über die Definition der Begrifflichkeiten nachdenken und diese schriftlich auf dem Arbeitsblatt festhalten. Danach sollen sie sich überlegen, wie man die Begriffe sortieren kann, so dass die Begrifflichkeiten einen Forschungsprozess vom Anfang bis zum Ende beschreiben. Die Reihenfolge soll notiert werden (zum Beispiel mit Nummerierung). Die Studierenden werden darauf hingewiesen, dass nicht alle Begriffe die gleiche Wertigkeit haben müssen und dass eventuell auch Begriffe fehlen. Sie werden gebeten, sich darüber klar zu werden, wo Wissenslücken und Unklarheiten bestehen.

Im Folgenden werden die Studierenden in Gruppen mit 3-5 Teilnehmer/innen aufgeteilt. In den Gruppen sollen sie ihre Ergebnisse und Fragen diskutieren und sich auf einen gemeinsamen Prozess einigen. Dazu erhalten sie erneut ein Arbeitsblatt, Schere und Klebestift, so dass sie in der Diskussion die einzelnen Begriffe bei Bedarf verschieben können. Wenn sich die Gruppe einig ist, soll das Ergebnis auf einem Flip-Chart Papier lesbar festgehalten werden. Es ist wichtig, dass sich die Gruppe darüber einigt, welche Wissenslücken und Unklarheiten bestehen. Sowohl das Ergebnis wie auch die offenen Fragen werden dann von allen Gruppen im Plenum kurz vorgestellt (Dauer pro Gruppe maximal 5 Minuten). Die Seminarleitung fasst die Klarheiten und Unklarheiten zusammen.

Erfahrungsgemäß sind nach dieser Gruppenarbeit die Studierenden sehr viel offener für einen Vortrag darüber wie Forschungsprozesse ablaufen, was welche Begriffe bedeuten, wie sinnvolle Abläufe geplant werden (vergleiche Abbildung 2). Wichtig ist, dass an entsprechender Stelle auf die Wissenslücken und Unklarheiten aus den Kleingruppen eingegangen werden.

Diese Übung kann dann sowohl darin münden, dass die Teilnehmer/innen an eigenen kleinen Forschungsprojekten arbeiten (wenn das ganze Seminar dieses Thema behandelt) als auch darin, dass anhand einer Forschungsfrage oder anhand einer kleinen Studie / eines Artikels alle Inhalte erklärt beziehungsweise gefestigt werden. Gleich wie es nach der Übung weitergeht: zur guten Verankerung empfiehlt es sich, die Inhalte zum Ende des Seminars in irgendeiner Form nochmals abzufragen/aufzuarbeiten.

Zeit: $\quad 120-180$ Minuten (je nachdem wo die Schwerpunkte im Seminar liegen und wie lang der Vortrag ist)

Material: je ein Arbeitsblatt mit Begrifflichkeiten für jede/n Teilnehmer/in sowie zusätzlich für jede Kleingruppe eines.

Pro Kleingruppe: Flipchartpapier, 1 Klebestift, 1 Schere und zwei Stifte, um auf dem Flipchartpapier zu schreiben.

Vortrag zum Thema (gegebenenfalls mittels Präsentation, aber in jedem Fall Klarheit darüber, was man selbst sagen möchte).

Sehr wichtig damit diese Übung gelingt und die Teilnehmenden offen sind für die meist unbekannten Inhalte ist, 


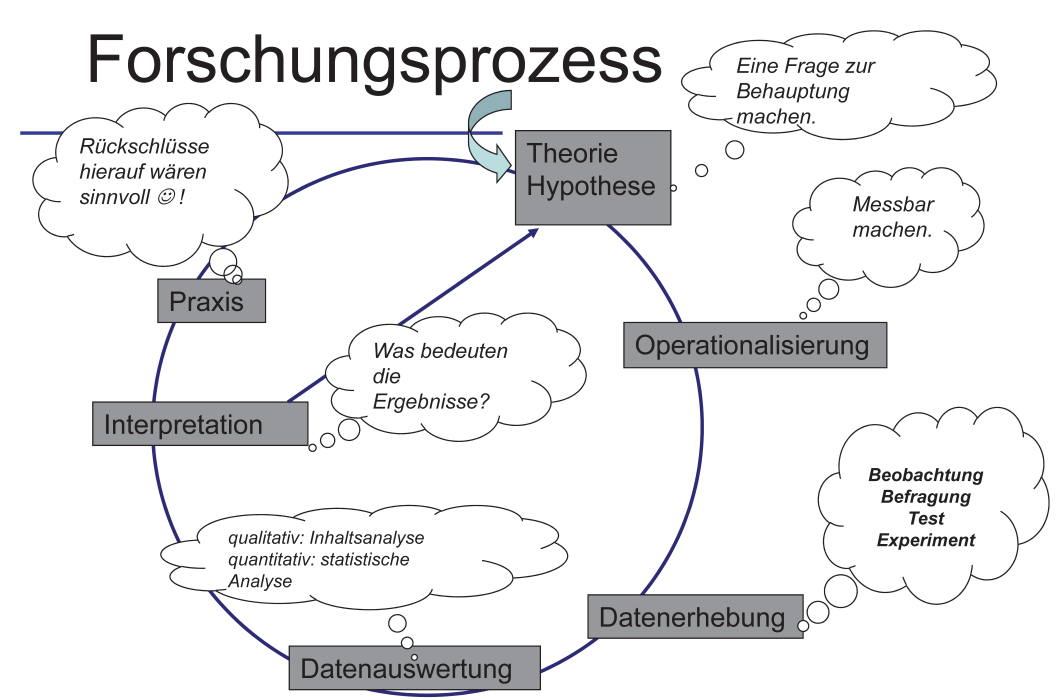

Abbildung 2.

dass in jedem Schritt $(1-3)$ sehr deutlich auf die Unklarheiten beziehungsweise offenen Fragen fokussiert wird. Während des gesamten Ablaufes ist die Seminarleitung im Raum und beantwortet Fragen, wenn sie dazu direkt angesprochen wird. Es ist nicht nachteilig, wenn einige Teilnehmer/innen schon genauere Vorstellungen von einigen Aspekten des Forschungsprozesses haben. Diese Teilnehmer/innen können im weiteren Verlauf aufgefordert werden, ihr Wissen weiterzugeben. Dies reduziert die Redezeit der Seminarleitung, trägt damit zu einer Aktivierung der gesamten Gruppe und mehr Interaktion bei und festigt das Wissen der Studierenden.

\section{Literatur}

Jürgen-Lohmann, J., Borsch, F. \& Giesen, H. (2001). Kooperatives Lernen an der Hochschule: Evaluation des Gruppenpuzzles in Seminaren der Pädagogischen Psychologie. Zeitschrift für Pädagogische Psychologie, 15(2), $74-84$.

Bortz, J. \& Döring, N. (2006). Forschungsmethoden und Evaluation für Human- und Sozialwissenschaftler. Heidelberg: Springer.

\section{Gedächtnis - Das Gedächtnismodell und Mnemotechniken}

Friederike Kendel, Anja Lehmann, Silke Burkert \& Juliane Wissmann

Kurzbeschreibung: Ausgehend von zwei Übungen sollen grundlegende Mechanismen der Gedächtnisbildung erläutert werden. Da die Quantität von Lerninhalten neue Lerntechniken im Medizinstudium fordert, sollen in den Übungen konkrete Lerninhalte aus dem medizinischen Curriculum (zum Beispiel aus Anatomie oder Hämatologie) verwendet werden.

\section{Lernziele}

Die Studierenden sollen ...

- die Komponenten des Gedächtnismodells (,Mehrspeichermodell“) benennen können.

- eine Mnemotechnik als eigene Lernstrategie anwenden können.

\section{Übung 1:}

Technische Voraussetzungen / Material: keine

Instruktion und Durchführung: Die Studierenden werden aufgefordert, sich im Raum umzusehen und zu zählen, wie viele Personen blaue Augen und wie viele Personen schwarze Haare haben. Die Augenfarbe kann auch per Handzeichen erhoben werden. Anschließend sollen alle Teilnehmer/innen die Augen schließen und die Frage beantworten, wie viele Personen im Raum (an diesem Tag) eine Brille tragen.

Auswertung: Erfahrungsgemäß schwanken die Zahlenangeben zu den Brillenträgern stark. Es ist wahrscheinlich, dass die Brillen wahrgenommen wurden, aber diese Information nicht über den sensorischen Speicher hinausgekommen ist. Das heißt, die Informationen haben den Weg vom sensorischen Speicher in das Kurz- oder Langzeitgedächtnis nicht gefunden. Während der Auswertung wird eine Folie zum Mehrspeichermodell des Gedächtnisses (Atkinson \& Shiffrin, 1968) gezeigt und es werden weitere Komponenten des Gedächtnismodells erläutert.

Zeit: $\quad 5-10 \mathrm{~min}$. 


\section{Übung 2:}

Technische Voraussetzungen / Material: zu memorierende Inhalte auf einer Folie (hier Beispiele aus Anatomie und Hämatologie)

Instruktion und Durchführung: Den Studierenden werden auf einer Folie Begriffe gezeigt wie zum Beispiel „Der Nervus accessorius innerviert den Musculus sternocleidomastoideus und den oberen Anteil des Musculus trapezius". Die Studierenden werden aufgefordert, zu den Nerven- und Muskelbezeichnungen möglichst fantasievolle Bilder zu finden. So könnte man sich ein Mädchen vorstellen, welches ein Sternenkleid trägt und über eine Rutschbahn zu einem Trapez gelangt.

Ein weiteres Beispiel für die assoziative Verknüpfung von Lerninhalten ist die Bildung von Eselsbrücken. So wäre „Never let monkeys eat bananas!“ ein Merksatz für weiße Blutzellen, die gleichzeitig nach ihrer Häufigkeit geordnet sind (Neutrophile $(60-70 \%)$, Lymphozyte (20-30\%), Monozyten (2-6\%), Eosinophile (1-5\%), Basophile $(<1 \%)$ ) (http://www.thieme.de/viamedici/lernen (eselsbruecken).

Auswertung: Namen und Bezeichnungen können besser erinnert werden, wenn sie mit Bildern, Eselsbrücken oder kleinen Geschichten verbunden werden. Je fantasievoller die Bilder sind, desto erfolgreicher können die Inhalte im Gedächtnis abgespeichert werden. Bei der Gedächtnisbildung arbeiten der ,visuelle Notizblock“ und die „,artikulatorische Schleife" unabhängig voneinander. Diese Tatsache kann man sich beim Lernen zunutze machen, indem man sowohl visuelle als auch verbale Strategien anwendet, das heißt sich Bilder vorstellt und gleichzeitig artikuliert. Praktisch alle Gedächtniskünstler nutzen verschiedene Sinnesorgane (-kanäle) und stellen sie in den Dienst der Gedächtnisbildung. Wichtig ist es, die Unabhängigkeit der beiden Speicher zu nutzen, statt sie gegeneinander arbeiten zu lassen. Also: Multitasking ja, aber beide Speicher in den Dienst der Sache stellen, da sonst der präfrontale Kortex überlastet wird. Je nach Lerntyp bietet es sich an, Bilder beziehungsweise Eselsbrücken gemeinsam zu generieren. Im Internet finden sich Seiten, auf denen Studierende gute Beispiele vorstellen (zum Beispiel http://www.thieme.de/viamedici/lernen/). Bei der Auswertung dieser Übung wird eine Folie des Arbeitsgedächtnismodells (Baddeley, 2000) gezeigt und diskutiert.

Dauer: $\quad 30-40 \mathrm{~min}$.

\section{Literatur}

Baddeley, A. D., Eysenck, M. W. \& Anderson, M. C. (2009). Memory. New York: Psychology Press.
Gluck, M. A., Mercado, E. \& Myers, C. E. (2010). Lernen und Gedächtnis. Vom Gehirn zum Verhalten. Heidelberg: Spektrum Akademischer Verlag.

http://www.thieme.de/viamedici/lernen/eselsbruecken. accessed: 3.10.2012

\section{Übung zur Sozialen Wahrnehmung „Hannelore und Maximilian“"}

Anja Fleischmann, Maike Lippmann, Stephanie Drössler, Andreas Dinkel \& Hendrik Berth

Kurzbeschreibung: Die Übung eignet sich als Einführung in das Thema "Soziale Wahrnehmung“ beziehungsweise „Wahrnehmung“. Veranschaulicht werden die Wirkmechanismen sozialer Wahrnehmung und die dabei automatisch ablaufenden Prozesse. Anhand einer kurzen Folienpräsentation erleben die Studierenden selbst, wie subjektive Faktoren wirksam werden.

Es ist möglich, diese Übung sowohl als „Aufhänger“ für eine Diskussion als auch als Einstieg in das Thema zu nutzen. Die Übung eignet sich zum Einsatz in Seminar oder Vorlesung, funktioniert mit minimalem Aufwand und kann deshalb variabel verwendet werden.

Instruktion und Durchführung: Die Studierenden werden in zwei Gruppen aufgeteilt (linke und rechte Seite). Beiden Gruppen werden abwechselnd auf Folien die Kurzbeschreibung einer Person (Geschlecht und persönliche Attribute, Krankheitssymptome) dargeboten (siehe Tabelle 1). Die Studierenden sollen aufgrund dieser wenigen Merkmale eine Einschätzung der Person sowie eine Einschätzung der Krankheitssymptome dieser Person vornehmen.

Die Ergebnisse werden von dem Dozierenden gesammelt, zusammengefasst und mit den Studierenden diskutiert. In der Zusammenfassung erscheint eine Auflösung der Folien (auf dieser stehen alle vorher präsentierten Merkmale im Überblick). So wird ersichtlich, dass bereits aufgrund weniger Merkmale ein komplexes Bild von einer Person entstehen kann, dass je nach Variation der Merkmale (hier nur geringe Variation) sehr unterschiedlich sein kann. Damit verbunden sind Urteilstendenzen/-fehler in der sozialen Wahrnehmung.

\section{Lernziele:}

Die Studierenden ....

- erläutern können, wie Wahrnehmung mit Prozessen der subjektiven Beurteilung und Bewertung assoziiert ist.

- werden für Prozesse der sozialen Wahrnehmung sensibilisiert. 
Tabelle 1: Anleitung für die Übung „Hannelore und Maximilian“ Anleitung für die Studierenden

Folie $1 \quad$ Bildung von zwei Studentengruppen (linke Hälfte/rechte Hälfte). In der Folge wird etwas präsentiert, das nur für die Studierenden der linken Hälfte beziehungsweise nur für die der rechten Hälfte gedacht ist. Wenn die Präsentation für die linke Hälfte läuft, schließen die Studierenden der rechten Hälfte bitte die Augen und umgekehrt.

Ihre Aufgabe: Lassen Sie die präsentierten Merkmale auf sich wirken und achten Sie dabei darauf, welche Vorstellung vor Ihrem inneren Auge entsteht.

Seien Sie bitte ruhig, lesen Sie die präsentierten Merkmale nicht laut vor, verraten Sie nicht, was Sie gelesen haben.

Die folgenden Folien werden abwechselnd präsentiert, dazwischen eignet sich eine leere Folie zum Wechsel der Gruppen (die linke Gruppe schließt jetzt die Augen, die rechte öffnet die Augen bzw. umgekehrt)

\begin{tabular}{|c|c|c|}
\hline & Linke Gruppe & Rechte Gruppe \\
\hline Folie 2/3 & $\begin{array}{l}\text { Ihre Arzthelferin sagt Ihnen, dass Ihr nächster Patient ein Mann } \\
\text { ist, der zum ersten Mal in Ihre Praxis kommt. }\end{array}$ & $\begin{array}{l}\text { Ihre Arzthelferin sagt Ihnen, dass Ihr nächster Patient eine Frau } \\
\text { ist, die zum ersten Mal in Ihre Praxis kommt. }\end{array}$ \\
\hline Folie $4 / 5$ & Der Patient ist Brillenträger. & Die Patientin ist Brillenträgerin. \\
\hline Folie $6 / 7$ & Der Patient ist attraktiv. & Die Patientin ist nicht attraktiv. \\
\hline \multirow[t]{2}{*}{ Folie 8/9 } & Der Vorname des Patienten ist Maximilian. & Der Vorname der Patientin ist Hannelore. \\
\hline & $\begin{array}{l}\text { Folie zur Zwischenmoderation (achten Sie darauf, dass die Stud } \\
\text { male nennen) }\end{array}$ & dierenden beim Antworten nicht die vorher präsentierten Merk- \\
\hline \multirow[t]{2}{*}{ Folie 10} & $\begin{array}{l}\text { Wie alt ist die Person? } \\
\text { Wie intelligent ist die Person? } \\
\text { Wie sympathisch ist Ihnen die Person? } \\
\text { Wegen welcher Beschwerden kommt Sie wohl zu Ihnen? }\end{array}$ & \\
\hline & Präsentieren Sie nun den Gruppen abwechselnd folgende beide & Folien \\
\hline \multirow[t]{2}{*}{ Folie $11 / 12$} & $\begin{array}{l}\text { Der Patient schildert als Beschwerden: } \\
\text { vermehrtes Schwitzen } \\
\text { Atemnot } \\
\text { Stechen und Ziehen in der Brust } \\
\text { Schwindel } \\
\text { verminderte Leistungsfähigkeit }\end{array}$ & $\begin{array}{l}\text { Die Patientin schildert als Beschwerden: } \\
\text { vermehrtes Schwitzen } \\
\text { Atemnot } \\
\text { Stechen und Ziehen in der Brust } \\
\text { Schwindel } \\
\text { verminderte Leistungsfähigkeit }\end{array}$ \\
\hline & $\begin{array}{l}\text { Folie zur Moderation / Fragen an die Gesamtgruppe (achten Sie } \\
\text { vorher präsentierten Merkmale nennen). }\end{array}$ & wieder darauf, dass die Studierenden beim Antworten nicht die \\
\hline \multirow[t]{2}{*}{ Folie 13} & $\begin{array}{l}\text { Woran leidet die Person? } \\
\text { Welche Diagnose würden Sie ihr geben? }\end{array}$ & \\
\hline & Alle Informationen werden zur Auflösung gemeinsam auf einer Fc & olie präsentiert: \\
\hline \multirow[t]{2}{*}{ Folie 14} & $\begin{array}{l}\text { männlich } \\
\text { Brillenträger } \\
\text { attraktiv } \\
\text { Maximilian }\end{array}$ & $\begin{array}{l}\text { weiblich } \\
\text { Brillenträgerin } \\
\text { nicht attraktiv } \\
\text { Hannelore }\end{array}$ \\
\hline & $\begin{array}{l}\text { Der Patient schildert als Beschwerden: } \\
\text { vermehrtes Schwitzen } \\
\text { Atemnot } \\
\text { Stechen und Ziehen in der Brust } \\
\text { Schwindel } \\
\text { verminderte Leistungsfähigkeit }\end{array}$ & $\begin{array}{l}\text { Die Patientin schildert als Beschwerden: } \\
\text { vermehrtes Schwitzen } \\
\text { Atemnot } \\
\text { Stechen und Ziehen in der Brust } \\
\text { Schwindel } \\
\text { verminderte Leistungsfähigkeit }\end{array}$ \\
\hline
\end{tabular}

Technische Voraussetzungen / Material: Für die Übung werden Computer, Beamer und die vorbereiteten Präsentations-Folien (Tabelle 1) benötigt. Gruppengröße: sowohl für Seminare (20 Personen) als auch Vorlesung geeignet.

Dauer: Zirka 15 min; als ,Aufhänger“ für eine längere Diskussion sollten 30-40 Minuten eingeplant werden.
Instruktion und Durchführung: Die Studierenden werden in zwei Gruppen eingeteilt (am einfachsten: rechte und linke Hälfte des Raumes). Den beiden Gruppen werden abwechselnd verschiedene Merkmale einer Person präsentiert, so dass vor dem geistigen Auge das Bild dieser Person entstehen kann. Die Präsentation erfolgt abwechselnd über Folien, die Gruppe, die die Merkmale jeweils nicht sehen darf, 
soll dabei die Augen schließen. Nach dem Präsentieren der Merkmale werden die Studierenden gebeten, die Person hinsichtlich Alter, Sympathie, Intelligenz und möglichen Krankheiten einzuschätzen. Am Ende erfolgt eine Auflösung (alle Merkmale werden auf einer Folie präsentiert) und eine Auswertung.

\section{Literatur}

Studien zur unterschiedlichen Wirkung von Attraktivität, Vornamen, sozioökonomischen Status etc. auf unsere Wahrnehmung bzw. Beurteilung von Personen. Einige der in den Artikeln untersuchten Attribute werden in der Übung eingesetzt:

Forgas, J. P. (1994). Soziale Interaktion und Kommunikation. Eine Einführung in die Sozialpsychologie (2. Aufl.). Weinheim: Psychologie Verlags Union.

Rudolph, U. \& Spörrle, M. (1999). Alter, Attraktivität und Intelligenz von Vornamen: Wortnormen für Vornamen im Deutschen. Zeitschrift für Experimentelle Psychologie, 46, 115 - 128.

Rudolph, U., Böhm, R \& Lummer, M. (2007). Ein Vorname sagt mehr als 1000 Worte - zur sozialen Wahrnehmung von Vornamen. Zeitschrift für
Sozialpsychologie, 38(1), $17-31$.

Woo, J. K. H., Ghorayeb, S.H., Lee, C. K., Sangha, H. \& Richter. S. (2004). Effect of patient socioeconomic status on perceptions of first- and second-year medical students. Canadian Medical Association Journal, 170, $1915-1919$.

\section{Ausblick}

Die hier vorgestellten Übungen haben sich in der medizinpsychologischen Lehre bereits bewährt. Da es insgesamt einen Mangel an Übungen gibt, mit denen ein guter Einstieg in komplexe Inhalte gelingt und mit denen theoretische Inhalte anschaulicher vermittelt werden können, soll mit diesen Übungen ein Anfang gemacht werden. Es ist zu hoffen, dass auf Dauer zu diesen und anderen Themen des medizinpsychologischen Curriculums weitere Beispiele zusammengetragen werden. 